\title{
CONFIRMATION OF A KINEMATIC DIAGNOSTIC FOR FACE-ON BOX/PEANUT-SHAPED BULGES ${ }^{1}$
}

\author{
J. Méndez-Abreu, ${ }^{2,3,4}$ E. M. Corsini, ${ }^{3}$ Victor P. Debattista, ${ }^{5,6}$ S. De Rijcke, ${ }^{7}$ J. A. L. Aguerri, ${ }^{8}$ And A. Pizzella ${ }^{3}$ \\ Received 2008 February 29; accepted 2008 April 18; published 2008 May 6
}

\begin{abstract}
We present the results of high-resolution absorption-line spectroscopy of three face-on galaxies, NGC 98, NGC 600 , and NGC 1703, with the aim of searching for box/peanut (B/P) shaped bulges. These observations test and confirm, for the first time, the prediction that face-on B/P-shaped bulges can be recognized by two minima in the profile along the bar's major axis of the fourth Gauss-Hermite moment $h_{4}$ of the line-of-sight velocity distribution (LOSVD). In NGC 98, a clear double minimum in $h_{4}$ is present along the major axis of the bar and before the end of the bar, as predicted. In contrast, in NGC 600, which is also a barred galaxy but lacks a substantial bulge, we do not find any significant kinematic signature for a B/P-shaped bulge. In NGC 1703, which is an unbarred control galaxy, we found no evidence of a $\mathrm{B} / \mathrm{P}$ bulge. We also show directly that the LOSVD is broader at the location of the $h_{4}$ minimum in NGC 98 than elsewhere. This more direct method avoids possible artifacts associated with the degeneracy between the measurement of LOSVD and $h_{4}$.
\end{abstract}

Subject headings: galaxies: bulges — galaxies: evolution — galaxies: formation galaxies: kinematics and dynamics — galaxies: spiral

\section{INTRODUCTION}

Roughly one-quarter of the visible light emitted by stars in the local universe comes out of the bulges of disk galaxies (Persic \& Salucci 1992; Fukugita et al. 1998). Understanding how bulges form is therefore of great importance to developing a complete picture of galaxy formation. The processes by which bulges form are still debated. On one hand, the merger of dwarfsized galactic subunits has been suggested as the main path for bulge formation (Kauffmann et al. 1993), which is supported by the relatively homogeneous bulge stellar populations of the Milky Way and M31 (Ferreras et al. 2003; Zoccali et al. 2003; Stephens et al. 2003). Bulges formed in such mergers are termed "classical" bulges. Alternatively, bulges may form via internal "secular" processes such as bar-driven gas inflows, bending instabilities, and clump instabilities (Combes \& Sanders 1981; Pfenniger 1984; Combes et al. 1990; Pfenniger \& Friedli 1991; Raha et al. 1991; Noguchi 1999; Immeli et al. 2004; Athanassoula 2005; Debattista et al. 2006). Moreover, the growth of the bulge out of disk material may also be externally triggered by satellite accretion during minor merging events (Aguerri et al. 2001; Eliche-Moral et al. 2006) or also via external "secular" processes. Evidence for secular bulge formation includes the near-exponential bulge light profiles (Andredakis \& Sanders 1994; Andredakis et al. 1995; Courteau et al. 1996; de Jong 1996; Carollo et al. 1998, 2001; Prieto et al. 2001; Carollo 1999; MacArthur et al. 2003), a correlation

\footnotetext{
1 Based on observations collected at the European Southern Observatory, Chile (ESO No. 76.B-0324(A)).

${ }^{2}$ INAF-Osservatorio Astronomico di Padova, vicolo dell'Osservatorio 5, I35122 Padova, Italy; jairo.mendez@oapd.inaf.it.

${ }^{3}$ Dipartimento Astronomia, Università di Padova, vicolo dell'Osservatorio 3 , I-35122 Padova, Italy; enricomaria.corsini@unipd.it, alessandro.pizzella@ unipd.it.

${ }^{4}$ Universidad de La Laguna, Avenida Astrofísico Francisco Sánchez s/n, E-38206 La Laguna, Spain.

${ }^{5}$ Centre for Astrophysics, University of Central Lancashire, Preston PR1 2HE, UK; vpdebattista@uclan.ac.uk.

${ }^{6}$ RCUK Fellow.

7 Sterrenkundig Observatorium, Universiteit Gent, Krijgslaan 281, S9, B9000 Gent, Belgium; sven.derijcke@ugent.be.

${ }^{8}$ Instituto de Astrofísica de Canarias, Calle Via Láctea s/n, E-38200 La Laguna, Spain; jalfonso@iac.es.
}

between bulge and disk scale lengths (de Jong 1996; MacArthur et al. 2003; Aguerri et al. 2005; Méndez-Abreu et al. 2008), the similar colors of bulges and inner disks (Terndrup et al. 1994; Peletier \& Balcells 1996; Courteau et al. 1996; Carollo et al. 2007), substantial bulge rotation (Kormendy 1993; Kormendy et al. 2002), and the presence of $\mathrm{B} / \mathrm{P}$-shaped bulges in $\sim 45 \%$ of edge-on disk galaxies (Lütticke et al. 2000). A review of secular "pseudobulge" formation and evidence for it can be found in Kormendy \& Kennicutt (2004). Standard cold dark matter cosmology predicts that galaxies without classical bulges should be rare (D'Onghia \& Burkert 2004). Not only are they not rare (e.g., Laurikainen et al. 2007), they are more prevalent among normal galaxies than classical bulges, exacerbating the disagreement between theory and observations (Debattista et al. 2006). It is therefore important to determine which bulges are of the classical versus pseudo variety, and which are a mix of both.

$\mathrm{N}$-body simulations show that barred galaxies have a tendency to develop B/P bulges (Combes \& Sanders 1981; Combes et al. 1990; Pfenniger \& Friedli 1991; Raha et al. 1991; Debattista et al. 2006). The fraction of edge-on galaxies with $\mathrm{B} / \mathrm{P}$ bulges is comparable to the fraction of disk galaxies containing bars ( 60\%; Eskridge et al. 2000; Marinova \& Jogee 2007) hinting that the two are related. The presence of bars in edge-on galaxies with $\mathrm{B} / \mathrm{P}$ bulges has been established by the kinematics of gas and stars (Kuijken \& Merrifield 1995; Merrifield \& Kuijken 1999; Bureau \& Freeman 1999; Bureau \& Athanassoula 1999, 2005; Vega Beltrán et al. 1997; Athanassoula \& Bureau 1999; Chung \& Bureau 2004). However, the degeneracy inherent in deprojecting edge-on galaxies makes it difficult to study other properties of the host galaxy. For example, simulations show that a bar can produce a $\mathrm{B} / \mathrm{P}$ shape even if a massive classical bulge formed before the disk (Athanassoula \& Misiriotis 2002; Debattista et al. 2005). Understanding the relative importance of classical and pseudobulges requires an attempt at a cleaner separation of bulges, bars, and peanuts, which is easiest to accomplish in less inclined systems. At least in two cases, NGC 4442 (Bettoni \& Galletta 1994) and NGC 7582 (Quillen et al. 1997), a B/P bulge can be recognized at inclination $i<75^{\circ}$, which permits also a direct identification of the bar. Debattista et al. (2005) proposed a kine- 
matic diagnostic of $\mathrm{B} / \mathrm{P}$ bulges which properly works up to a disk inclination of about $30^{\circ}$, namely, a double minimum in the fourth moment, $h_{4}$, of the line-of-sight velocity distribution (LOSVD), along the major-axis of the bar. These minima occur because at the location of the $\mathrm{B} / \mathrm{P}$ shape, the vertical density distribution of stars becomes broader, which leads to a double minimum in $z_{4}$, the fourth-order Gauss-Hermite moment of the vertical density distribution. The kinematic moment $h_{4}$ is then found to be an excellent proxy for the unobservable $z_{4}$. In contrast, the increase in the vertical scale height does not produce any distinct signature of a $\mathrm{B} / \mathrm{P}$ bulge and the vertical velocity dispersion, $\sigma_{z}$, is too strongly dependent on the radial density variation to provide a useful $\mathrm{B} / \mathrm{P}$ bulge diagnostic. Debattista et al. (2006) showed that the diagnostic continues to hold even when gas is present since this sinks to a radius smaller than that of the $\mathrm{B} / \mathrm{P}$ bulge.

In this Letter we present the first high-resolution stellar kinematics of face-on galaxies with the goal of searching for B/ $\mathrm{P}$-shaped bulges and testing the diagnostic proposed by Debattista et al. (2005). In $\S 2$ we describe the observations of the sample and data reduction, while our results are presented in $\S 3$. Finally, the conclusions are given in $\S 4$.

\section{OBSERVATIONS AND DATA REDUCTION}

\subsection{Sample Selection}

The barred galaxies NGC 98 and NGC 600 were selected in the NASA/IPAC Extragalactic Database (NED) as bright $\left(B_{T}<14\right)$ and undisturbed objects, with a disk inclination lower than $30^{\circ}$ and a bar length larger than $10^{\prime \prime}$ to efficiently test the kinematic diagnostic, a disk diameter smaller than $4^{\prime}$ to allow an accurate sky subtraction, no strong evidence of dust, and no bright foreground stars in the Digitized Sky Survey image. To select the sample galaxies, their disk inclination, bar length, and bar position angle were first determined from ellipse fits to the 2MASS $J$-band archival images. The main properties of the sample galaxies are given in Table 1 .

\subsection{Long-Slit Spectroscopy}

The spectroscopic observations were carried out in service mode at the Very Large Telescope (VLT) at the European Southern Observatory (ESO) on 2005 October 2-9 and November 2. The Focal Reducer Low Dispersion Spectrograph 2 (FORS2) mounted the volume-phased holographic grism GRIS_1028z+29 with 1028 grooves $\mathrm{mm}^{-1}$ and the $0.7^{\prime \prime} \times 6.8^{\prime \prime}$ slit. The detector was a mosaic of 2 MIT/LL CCDs, each with $2048 \times 2068$ pixels of $15 \times 15 \mu \mathrm{m}^{2}$. The wavelength range from 7681 to $9423 \AA$ was covered in the reduced spectra with a reciprocal dispersion of $0.858 \AA$ pixel $^{-1}$ and a spatial scale of $0.250^{\prime \prime}$ pixel $^{-1}$ after a $2 \times 2$ pixel binning. The spectra were taken along the bar major axis of NGC 98 (P.A. $=32.2^{\circ}$ ) and NGC 600 (P.A. $=22.1^{\circ}$ ) and along the disk major axis of NGC 1703 (P.A. $=148.8^{\circ}$ ). The total integration time for each galaxy was $3 \mathrm{hr}$, in four exposures of 45 minutes each. Comparison lamp exposures obtained for each observing night ensured accurate wavelength calibrations. Spectra of $\mathrm{G}$ and $\mathrm{K}$ giant stars served as kinematic templates. The average seeing FWHM was $1.3^{\prime \prime}$ for NGC 98, 0.9" for NGC 600, and $0.9^{\prime \prime}$ for NGC 1703 as measured from the ESO Differential Imaging Meteo Monitor.

Using standard $\operatorname{IRAF}^{9}$ routines, all the spectra were bias

\footnotetext{
${ }^{9}$ IRAF is distributed by NOAO, which is operated by AURA, Inc., under contract with the National Science Foundation.
}

TABLE 1

Parameters of the Sample Galaxies

\begin{tabular}{clccccc}
\hline \hline Object & Type & $B_{T}$ & $\begin{array}{c}D_{25} \\
(\operatorname{arcmin})\end{array}$ & $M_{B_{T}}^{0}$ & $\begin{array}{c}i \\
(\mathrm{deg})\end{array}$ & $\begin{array}{c}a_{B} \\
(\operatorname{arcsec})\end{array}$ \\
\hline NGC $98 \ldots \ldots \ldots$ & SBbc & 13.4 & 1.7 & -22.4 & 26 & $14 \pm 1$ \\
NGC $600 \ldots \ldots \ldots$ & SBd & 12.9 & 3.3 & -20.0 & 21 & $12 \pm 1$ \\
NGC $1703 \ldots \ldots$ & Sc & 11.9 & 3.0 & -20.6 & 15 & $\ldots$ \\
\hline
\end{tabular}

Notes. - Hubble types, apparent magnitudes, and diameters are from the NASA/IPAC Extragalactic Database (NED). Absolute magnitudes are calculated from $B_{T}^{0}$ (NED) with distance from $V_{\mathrm{CMB}}$ (NED) and $H_{0}=75 \mathrm{~km} \mathrm{~s}^{-1}$ $\mathrm{kpc}^{-1}$. Disk inclinations and bar semimajor axis lengths are from this Letter.

subtracted, flat-field corrected, cleaned of cosmic rays, corrected for bad pixels, and wavelength calibrated as in Debattista et al. (2002). The accuracy of the wavelength rebinning $(\approx 1$ $\mathrm{km} \mathrm{s}^{-1}$ ) was checked by measuring wavelengths of the brightest night-sky emission lines. The instrumental resolution was $1.84 \pm 0.01 \AA(\mathrm{FWHM})$ corresponding to $\sigma_{\text {inst }}=27 \mathrm{~km} \mathrm{~s}^{-1}$ at $8552 \AA$. The spectra obtained for the same galaxy were coadded using the center of the stellar continuum as reference. In the resulting spectra the sky contribution was determined by interpolating along the outermost $\approx 30^{\prime \prime}$ at the edges of the slit and then subtracted.

\subsection{Photometry}

We analyzed the uncalibrated acquisition images from the VLT to derive the photometric properties of the sample galaxies. Isophote-fitting with ellipses, after masking foreground stars and bad pixels, was carried out using the IRAF task ELLIPSE. Under the assumption that the outer disks are circular, their inclination was determined by averaging the outer isophotes. All three galaxies have $i<30^{\circ}$ (Table 1). The semimajor axis length, $a_{B}$, of the bars of NGC 98 and NGC 600 (Table 1) was measured from a Fourier decomposition as in Aguerri et al. (2000). It was calculated using the bar/interbar intensity ratio $I_{b} / I_{\mathrm{ib}}$. The bar intensity is defined as the sum of the even Fourier components, $I_{0}+I_{2}+I_{4}+I_{6}$, while the interbar intensity is given by $I_{0}-I_{2}+I_{4}-I_{6}$. The bar length is the FWHM of radial profile of $I_{b} / I_{\mathrm{ib}}$. This method was applied by Athanassoula \& Misiriotis (2002) to analytic models demonstrating its accuracy in measuring $a_{B}$.

\subsection{Kinematics}

The stellar kinematics of the three galaxies were measured from the galaxy absorption features present in the wavelength range centered on the Ca II triplet $(\lambda \lambda 8498,8542,8662 \AA)$ using the penalized pixel-fitting method (pPXF; Cappellari \& Emsellem 2004). The spectra were rebinned along the dispersion direction to a logarithmic scale, and along the spatial direction to obtain a signal-to-noise ratio $\mathrm{S} / \mathrm{N} \gtrsim 40 \AA^{-1}$. It decreases to $\mathrm{S} / \mathrm{N}=20 \AA^{-1}$ at the outermost radii.

A linear combination of the template stellar spectra, convolved with the line-of-sight velocity distribution (LOSVD) described by the Gauss-Hermite expansion by van der Marel \& Franx (1993) was fitted to each galaxy spectrum by $\chi^{2}$ minimization in pixel space. This allowed us to derive profiles of the mean velocity $\left(v_{\text {los }}\right)$, velocity dispersion $\left(\sigma_{\text {los }}\right)$, and third$\left(h_{3}\right)$ and fourth-order $\left(h_{4}\right)$ Gauss-Hermite moments. The uncertainties on the kinematic parameters were estimated by Monte Carlo simulations with photon, readout, and sky noise. Extensive testing on simulated galaxy spectra was performed to provide an estimate of the biases of the pPXF method with the adopted instrumental setup and spectral sampling. The simulated spectra were obtained by convolving the template spectra 

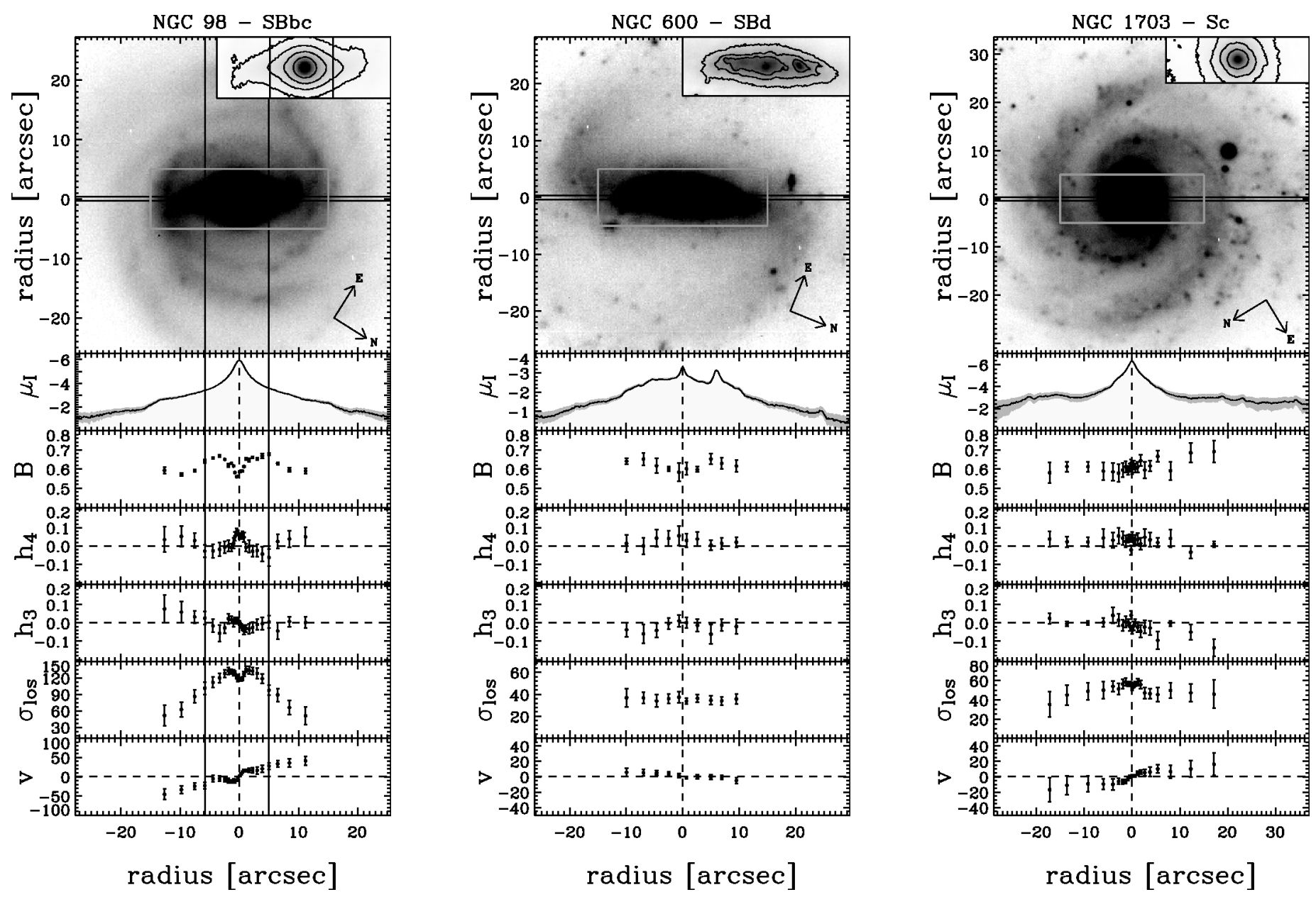

FIG. 1.-Morphology and stellar kinematics of NGC 98 (left panels), NGC 600 (central panels), and NGC 1703 (right panels). For each galaxy the top panel shows the VLT FORS2 I-band image. The slit position and image orientation are indicated. The inset shows the portion of the galaxy image marked with a white box. The gray scale and isophotes were chosen to enhance the features observed in the central regions. The remaining panels show from top to bottom the radial profiles of surface brightness (extracted along the slit with an arbitrary zero point), $\mathcal{B}(0.7,0.9), h_{4}, h_{3}, \sigma_{\text {los }}$ (in $\mathrm{km} \mathrm{s}^{-1}$ ), and velocity $v$ (in $\mathrm{km} \mathrm{s}^{-1}$; obtained by subtracting the systemic velocity from $v_{\text {los }}$ ). The two vertical lines indicate the location of the $h_{4}$ minima in NGC 98 .

with a LOSVD parameterized as a Gauss-Hermite series and measured as if they were real. No bias was found in the ranges of $\mathrm{S} / \mathrm{N}$ and $\sigma_{\text {los }}$ which characterize the spectra of the sample galaxies. The values of $h_{3}$ and $h_{4}$ measured for the simulated spectra differ from the intrinsic ones only within the measured errors (see also Emsellem et al. 2004).

\section{RESULTS}

The photometric and kinematic profiles of the sample of galaxies are shown in Figure 1. We focus here on the $h_{4}$ profiles; a full analysis of this data set will be presented in a forthcoming paper. For the unbarred galaxy, NGC 1703, the $h_{4}$ profile shows no sign of a minimum. The same is true for the almost bulgeless, barred NGC 600. In both these galaxies $\sigma>30 \mathrm{~km}$ $\mathrm{s}^{-1}$, including within the bar in NGC 600. Thus, our failure to find an $h_{4}$ minimum cannot be ascribed to low spectral resolution. In NGC 98, instead, we find a double minimum in the $h_{4}$ profile. The two minima are symmetric with respect to the galaxy center $\left(|r| \approx 5^{\prime \prime}\right)$. In the simulations of Debattista et al. (2005) the minima in $h_{4}$ are $\sim 0.05-0.1$ deep, comparable to the minimum in the profile of NGC 98.

We confirmed that our kinematics, and minima in $h_{4}$, are not a result of details of the pPXF method by repeating the analysis using the Fourier correlation quotient (FCQ) method (Bender 1990) as done in Pizzella et al. (2008). Nonetheless, expansions in Gauss-Hermite moments are degenerate (Gerhard 1993; van der Marel \& Franx 1993) since, for the even moments, it is possible to obtain comparably good fits by trading $\sigma_{\text {los }}$ for $h_{4}$. In order to confirm that the signature of a $\mathrm{B} / \mathrm{P}$ bulge obtained in NGC 98 is not due to a conspiracy of such degeneracies we introduced an LOSVD broadening measure defined for each radius as

$$
\mathcal{B}\left(x_{1}, x_{2}\right) \equiv W\left(x_{2}\right) / W\left(x_{1}\right)
$$

where

$$
W(x)=\int_{v_{\mathrm{los}}^{\min }}^{v_{\mathrm{fos}}^{\max }} F(v) d v
$$

with $F(v)$ the cumulative distribution of the LOSVD. The values of $v_{\mathrm{los}}^{\mathrm{min}}$ and $v_{\mathrm{los}}^{\max }$ correspond to $F\left(v_{\mathrm{los}}^{\mathrm{min}}\right)=0.5(1-x)$ and $F\left(v_{\text {los }}^{\max }\right)=0.5(1+x)$, respectively. $\mathcal{B}\left(x_{1}, x_{2}\right)$ has the advantage of not being sensitive to the wings of the distribution, unlike the kurtosis, and is a nonparametric measure of the LOSVD shape. Experiments with $N$-body simulations, including those presented in Debattista et al. (2005), showed that $\mathcal{B}(0.7,0.9)$ is a useful peanut diagnostic in the same spirit as $h_{4}$. Figure 1 plots $\mathcal{B}(0.7,0.9)$. A clear peak in the broadening is apparent 
at $|r| \simeq 5^{\prime \prime}$ in NGC 98. We conclude that the evidence for a $\mathrm{B} / \mathrm{P}$-shaped bulge in NGC 98 is robust.

\section{CONCLUSIONS}

We have identified a $\mathrm{B} / \mathrm{P}$-shaped bulge in one galaxy from a sample of three face-on galaxies. In the unbarred control galaxy NGC 1703 we had not expected to find a B/P bulge. The failure to find a double minimum in $h_{4}$ is therefore fully consistent with previous results (Chung \& Bureau 2004). Of the two barred galaxies, NGC 98 has clear evidence of a B/P bulge while NGC 600 does not. The absence of a B/P shape in the latter galaxy is not surprising since it appears to not have a bulge and its bar has a lumpy structure.

If we identify the radius of the $\mathrm{B} / \mathrm{P}$ bulge, $R_{\mathrm{B} / \mathrm{P}}$, with the location of the minimum in $h_{4}$, as found in simulations (Debattista et al. 2005), then we find $R_{\mathrm{B} / \mathrm{P}} \simeq 0.35 a_{B}$, where $a_{B}$ is the bar semimajor axis. Similarly, Kormendy \& Kennicutt (2004) noted that the maximum radius of the boxy bulge is about one-third of the bar radius. Simulations also produce B/ P-bulges which are generally smaller than the bar (Shen \& Sellwood 2004; Debattista et al. 2005).
While observations have established the presence of bars in the majority of systems with B/P-bulges, the converse has not been established. Debattista et al. (2005) demonstrate how the presence of a classical bulge can in some cases mask the presence of $\mathrm{B} / \mathrm{P}$ bulge. Moreover, the ratio of radii of bulges and $\mathrm{B} / \mathrm{P}$ bulges is determined by resonances. For these various reasons it would be very instructive to repeat measurements such as here for a sample of barred galaxies.

We thank Eva Grebel for her support and Lorenzo Morelli for fruitful discussion. E. M. C. and V. P. D. thank the Instituto de Astrofísica de Canarias for hospitality during part of this project. J. M. A. acknowledges support from the Istituto nazionale di Astrofisica (INAF). E. M. C. and A. P. receive support from the grant CPDA068415/06 by Padua University. S. D. R. acknowledges postdoctoral fellowship support from the Fund for Scientific Research-Flanders, Belgium (FWO). J. A. L. A. is funded by the Spanish DGES, grant AYA-2007-67965C03-01. This research has made use of the NASA/IPAC Extragalactic Database, Digitized Sky Survey, and 2 Micron All Sky Survey.

\section{REFERENCES}

Aguerri, J. A. L., Balcells, M., \& Peletier, R. F. 2001, A\&A, 367, 428

Aguerri, J. A. L., Elias-Rosa, N., Corsini, E. M., \& Muñoz-Tuñón, C. 2005, A\&A, 434, 109

Aguerri, J. A. L., Muñoz-Tuñón, C., Varela, A. M., \& Prieto, M. 2000, A\&A, 361,841

Andredakis, Y. C., Peletier, R. F., \& Balcells, M. 1995, MNRAS, 275, 874

Andredakis, Y. C., \& Sanders, R. H. 1994, MNRAS, 267, 283

Athanassoula, E. 2005, MNRAS, 358, 1477

Athanassoula, E., \& Bureau, M. 1999, ApJ, 522, 699

Athanassoula, E., \& Misiriotis, A. 2002, MNRAS, 330, 35

Bender, R. 1990, A\&A, 229, 441

Bettoni, D., \& Galletta, G. 1994, A\&A, 281, 1

Bureau, M., \& Athanassoula, E. 1999, ApJ, 522, 686 2005, ApJ, 626, 159

Bureau, M., \& Freeman, K. C. 1999, AJ, 118, 126

Cappellari, M., \& Emsellem, E. 2004, PASP, 116, 138

Carollo, C. M. 1999, ApJ, 523, 566

Carollo, C. M., Scarlata, C., Stiavelli, M., Wyse, R. F. G., \& Mayer, L. 2007, ApJ, 658, 960

Carollo, C. M., Stiavelli, M., de Zeeuw, P. T., Seigar, M., \& Dejonghe, H. 2001, ApJ, 546, 216

Carollo, C. M., Stiavelli, M., \& Mack, J. 1998, AJ, 116, 68

Chung, A., \& Bureau, M. 2004, AJ, 127, 3192

Combes, F., Debbasch, F., Friedli, D., \& Pfenniger, D. 1990, A\&A, 233, 82

Combes, F., \& Sanders, R. H. 1981, A\&A, 96, 164

Courteau, S., de Jong, R. S., \& Broeils, A. H. 1996, ApJ, 457, L73

Debattista, V. P., Carollo, C. M., Mayer, L., \& Moore, B. 2005, ApJ, 628, 678

Debattista, V. P., Corsini, E. M., \& Aguerri, J. A. L. 2002, MNRAS, 332, 65

Debattista, V. P., Mayer, L., Carollo, C. M., Moore, B., Wadsley, J., \& Quinn, T. 2006, ApJ, 645, 209

de Jong, R. S. 1996, A\&A, 313, 45

D’Onghia, E., \& Burkert, A. 2004, ApJ, 612, L13

Eliche-Moral, M. C., Balcells, M., Aguerri, J. A. L., \& González-García, A. C. 2006, A\&A, 457, 91

Emsellem, E., et al. 2004, MNRAS, 352, 721

Eskridge, P. B., et al. 2000, AJ, 119, 536

Ferreras, I., Wyse, R. F. G., \& Silk, J. 2003, MNRAS, 345, 1381

Fukugita, M., Hogan, C. J., \& Peebles, P. J. E. 1998, ApJ, 503, 518

Gerhard, O. E. 1993, MNRAS, 265, 213
Immeli, A., Samland, M., Gerhard, O., \& Westera, P. 2004, A\&A, 413, 547

Kauffmann, G., White, S. D. M., \& Guiderdoni, B. 1993, MNRAS, 264, 201

Kormendy, J. 1993, in IAU Symp. 153, Galactic Bulges, ed. H. Dejonghe \& H. J. Habing (Dordrecht: Kluwer), 209

Kormendy, J., Bender, R., \& Bower, G. 2002, in ASP Conf. Ser. 273, Dynamics, Structure, and History of Galaxies, ed. G. S. Da Costa \& H. Jerjen (San Francisco: ASP), 29

Kormendy, J., \& Kennicutt, R. C., Jr. 2004, ARA\&A, 42, 603

Kuijken, K., \& Merrifield, M. R. 1995, ApJ, 443, L13

Laurikainen, E., Salo, H., Buta, R., \& Knapen, J. H. 2007, MNRAS, 381, 401

Lütticke, R., Dettmar, R.-J., \& Pohlen, M. 2000, A\&AS, 145, 405

MacArthur, L. A., Courteau, S., \& Holtzman, J. A. 2003, ApJ, 582, 689

Marinova, I., \& Jogee, S. 2007, ApJ, 659, 1176

Méndez-Abreu, J., Aguerri, J. A. L., Corsini, E. M., \& Simonneau, E. 2008, A\&A, 478, 353

Merrifield, M. R., \& Kuijken, K. 1999, A\&A, 345, L47

Noguchi, M. 1999, ApJ, 514, 77

Peletier, R. F., \& Balcells, M. 1996, AJ, 111, 2238

Persic, M., \& Salucci, P. 1992, MNRAS, 258, 14

Pfenniger, D. 1984, A\&A, 134, 373

Pfenniger, D., \& Friedli, D. 1991, A\&A, 252, 75

Pizzella, A., Corsini, E. M., Sarzi, M., Magorrian, J., Méndez-Abreu, J., Coccato, L., Morelli, L., \& Bertola, F. 2008, MNRAS, in press (arXiv: 0803.2689)

Prieto, M., Aguerri, J. A. L., Varela, A. M., \& Muñoz-Tuñón, C. 2001, A\&A, 367,405

Quillen, A. C., Kuchinski, L. E., Frogel, J. A., \& Depoy, D. L. 1997, ApJ, 481, 179

Raha, N., Sellwood, J. A., James, R. A., \& Kahn, F. D. 1991, Nature, 352, 411

Shen, J., \& Sellwood, J. A. 2004, ApJ, 604, 614

Stephens, A. W., et al. 2003, AJ, 125, 2473

Terndrup, D. M., Davies, R. L., Frogel, J. A., Depoy, D. L., \& Wells, L. A. 1994, ApJ, 432, 518

van der Marel, R. P., \& Franx, M. 1993, ApJ, 407, 525

Vega Beltrán, J. C., Corsini, E. M., Pizzella, A., \& Bertola, F. 1997, A\&A, 324,485

Zoccali, M., et al. 2003, A\&A, 399, 931 